\title{
Breeding of Phenylalanine-producing Brevibacterium flavum Strains by Removing Feedback Regulation of Both the Two Key Enzymes in Its Biosynthesis
}

\author{
Isamu Shiı, Shin-ichi Sugimoto and Kazue Kawamura \\ Central Research Laboratories, Ajinomoto Co., Inc., \\ Kawasaki-ku, Kawasaki, Kanagawa 210, Japan
}

Received March 22, 1988

\begin{abstract}
The growth of a $m$ FP-resistant Brevibacterium flavum mutant, No. 221-43, having $\mathrm{PDT}^{\mathrm{R}}$ was synergistically and completely inhibited by $m$ FP plus Tyr-Glu, but not by $m \mathrm{FP}$ plus tyrosine or $p \mathrm{FP}$ plus Tyr-Glu, whereas that of a mutant having $D^{\mathbf{R}}$ was only partially inhibited by $m$ FP plus TyrGlu. Tyr-Glu could replace tyrosine required for the growth of a tyrosine auxotroph. The phenylalanine uptake was competitively inhibited by tyrosine and the tyrosine uptake by phenylalanine. The phenylalanine uptake was also inhibited by $m F P$, but not by Tyr-Glu. Mutants having both $\mathrm{PDT}^{\mathrm{R}}$ and $\mathrm{DS}^{\mathrm{R}}$ derived from strain No. 221-43 were effectively selected by the resistance to $m \mathrm{FP}$ plus Tyr-Glu, and produced much larger amounts of phenylalanine, with small amounts of tyrosine, than the parent. By the same method, mutants having $\mathrm{DS}^{\mathrm{R}}$ and $\mathrm{PDT}^{\mathrm{R}}$, which produced $23.4 \mathrm{~g} / \mathrm{l}$ of phenylalanine at maximum, were obtained from a $p$ FP-resistant tyrosine auxotroph having PDT $^{\mathrm{R}}$ which produced $18 \mathrm{~g} / \mathrm{l}$. Similar mutants were also obtained from a tryptophan-producing strain, but produced smaller amounts of tryptophan than the parent, whereas the total amounts of tryptophan and phenylalanine produced were increased.
\end{abstract}

Brevibacterium flavum has two feedback regulations in its phenylalanine biosynthetic pathway, the synergistic inhibition of DS by phenylalanine and tyrosine, ${ }^{1)}$ and the inhibition of PDT by phenylalanine ${ }^{23}$ (Fig. 1). As reported in the previous paper, ${ }^{3)}$ mutants resistant to one of the feedback inhibitions were obtained as phenylalanine analogue (e.g., mFP)-resistant mutants. Mutants having $\mathrm{DS}^{\mathrm{R}}$ derived from the wild strain accumulated both phenylalanine and tyrosine, while those having $\mathrm{PDT}^{\mathrm{R}}$ accumulated only phenylalanine. ${ }^{3)}$ Whereas mutants having both $\mathrm{DS}^{\mathrm{R}}$ and $\mathrm{PDT}^{\mathrm{R}}$ seemed to be better phenylalanine producers, it was difficult to obtain them, because the mutants having either $\mathrm{DS}^{\mathrm{R}}$ or $\mathrm{PDT}^{\mathrm{R}}$ were already resistant to phenylalanine analogues. Furthermore, a preliminary experiment show- ed that the sensitivities to $m \mathrm{FP}$ of mutants having PDT $^{\mathrm{R}}$ and feedback-sensitive DS were not increased by the further addition of tyrosine, which stimulated the $m \mathrm{FP}$ inhibition of DS. ${ }^{1)}$ This seems to be due to competition between $m F P$ and tyrosine in the transport system common to aromatic amino acids. ${ }^{4}$ Sato et al. ${ }^{5}$ have shown that in the presence of $p \mathrm{FP}$, a phenylalanine analogue, tyrosine auxotrophs of Brevibacterium lactofermentum did not grow with tyrosine but did with a tyrosinecontaining dipeptide, Tyr-Glu. This suggests that Tyr-Glu was taken up without competition with $p \mathrm{FP}$ and tyrosine was generated inside the cells through its hydrolysis. Thus, it is likely that the addition of $m \mathrm{FP}$ and Tyr-Glu to the medium may increase the intracellular concentrations of both $m \mathrm{FP}$ and tyrosine enough

Abbreviations: DAHP, 3-deoxy-D-arabino-heptulosonate-7-phosphate; DS, DAHP synthase; PDT, prephenate dehydratase; CM, chorismate mutase; $\mathrm{DS}^{\mathrm{R}}$, DS resistant to feedback inhibition; PDT $^{\mathrm{R}}$, PDT resistant to feedback inhibition; $m \mathrm{FP}, m$-fluorophenylalanine; $p \mathrm{FP}, p$-fluorophenylalanine; Tyr-Glu, tyrosylglutamate; Tyr-Ala, tyrosylalanine; Phe-Ala, phenylalanylalanine; TES, $N$-tris(hydroxymethyl)methyl-aminoethane sulfonic acid. 


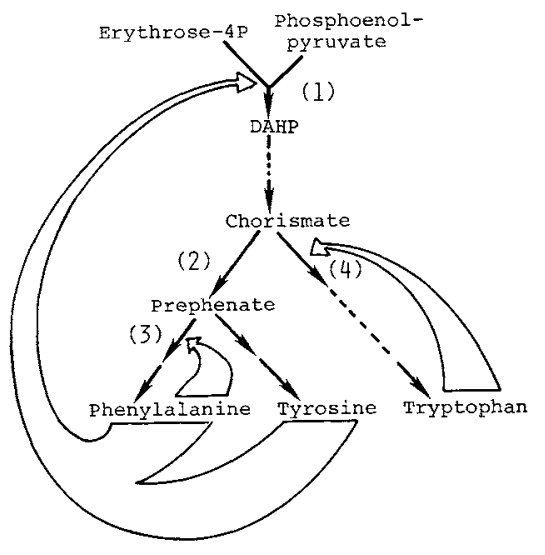

FIG. 1. Regulation of Aromatic Amino Acid Biosynthesis in Brevibacterium flavum.

$\Longrightarrow$, feedback inhibition; (1), DS; (2), CM; (3), PDT; (4), anthranilate synthase.

for them to inhibit DS synergistically, and, therefore, the growth of mutants having feedback-sensitive DS.

This paper reports confirmation of the suggestions described above, development of a method for the selection of mutants having both $\mathrm{DS}^{\mathrm{R}}$ and $\mathrm{PDT}^{\mathrm{R}}$ with $m \mathrm{FP}$-resistance in the presence of Tyr-Glu among those derived from strains having only $\mathrm{PDT}^{\mathrm{R}}$, and application of this method to the breeding of phenylalanine producers and tryptophan producers.

\section{MATERIALS AND METHODS}

Organisms. Brevibacterium flavum No. 2247 (ATCC 14067) and its mFP-resistant mutants, ${ }^{3)}$ No. 221-43 having $\mathrm{PDT}^{\mathrm{R}}$ and No. $482-18$ having $\mathrm{DS}^{\mathrm{R}}$, were used. Strain No. 239 is a phenylalanine-producing $p$ FP-resistant tyrosine-methionine-double auxotroph. ${ }^{6.71}$ Tryptophanproducing sulfaguanidine-resistant mutant S-179 was derived from strain A-100, which was an azaserine- and 5fluorotryptophan-resistant mutant derived from strain No. $239.6,8)$

Media. The compositions of the glucose-saits media, Medium $20,{ }^{97} 30,{ }^{10)}$ and $41,{ }^{11)}$ and complex medium Medium $7^{12)}$ were given in the previous papers. The following media were used for amino acid production, Medium 3, ${ }^{13)}$ containing $100 \mathrm{~g} / 1$ of glucose as a carbon source, and Medium $31,{ }^{10)}$ containing $130 \mathrm{~g}$ of glucose, $12 \mathrm{~g}$ of fumaric acid, and $3 \mathrm{ml}$ of acetic acid per liter.

Chemicals. DL- $m$ FP, DL-pFP, L-Tyr-L-Glu, L-Tyr-L-Ala, and L-Phe-L-Ala were purchased from Sigma Chemical Co. $\mathrm{L}-\left[\mathrm{U}-{ }^{14} \mathrm{C}\right] \mathrm{Phenylalanine}$ and $\mathrm{L}-\left[\mathrm{U}-{ }^{14} \mathrm{C}\right]$ tyrosine were from Amersham International plc.

Enzyme preparation. mFP-Resistant mutants derived from strain No. 221-43, strain S-179, and strain No. 239 were cultured at $30^{\circ} \mathrm{C}$ for $24 \mathrm{hr}$ in Medium 3, Medium 31 , and Medium 31 but containing $400 \mathrm{mg} / 1$ of L-tyrosine, respectively. The cells were harvested, washed twice with $0.2 \% \mathrm{KCl}$, suspended in $0.1 \mathrm{M}$ TES-NaOH buffer, $\mathrm{pH} 7.0$, containing $5 \mathrm{~mm} \mathrm{MgCl}, 2 \mathrm{~mm}$ dithiothreitol and $1 \mathrm{~mm}$ tryptophan, and then sonically disrupted at $10 \mathrm{kc}$ for $20 \mathrm{~min}$. The supernatant solutions of the sonicates obtained on centrifugation at $32,000 \times g$ for $30 \mathrm{~min}$ were gelfiltered on a Sephadex G-25 column $(1.5 \times 10 \mathrm{~cm})$ and then used as the enzymes. The buffers used for equilibration of the column were $0.05 \mathrm{M}$ TES-NaOH buffer, $\mathrm{pH} 7.0$, containing $5 \mathrm{~mm} \mathrm{MgCl}_{2}, 1 \mathrm{~mm}$ dithiothreitol, and $1 \mathrm{~mm}$ tryptophan for assaying DS, $0.05 \mathrm{~m}$ TES-NaOH buffer, $\mathrm{pH}$ 7.0, containing $5 \mathrm{mM} \mathrm{MgCl}$, for assaying $\mathrm{CM}$, and $0.05 \mathrm{M}$ Tris- $\mathrm{HCl}$ buffer, $\mathrm{pH} 7.5$, for assaying PDT.

Assay methods. The assay methods for DS, ${ }^{1 /} \mathrm{CM},{ }^{14)}$ and $\mathrm{PDT}^{2)}$ were described previously. The uptake of phenylalanine or tyrosine was assayed by a modification of the method described previously ${ }^{8}$ : strain No. 2247 was cultured at $30^{\circ} \mathrm{C}$ for $16 \mathrm{hr}$ in Medium 20 . The standard assay

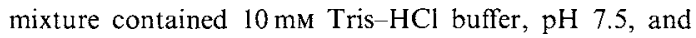
$0.05 \mathrm{ml}$ of a suspension of the washed cells (absorbancy at $562 \mathrm{~nm}$ after 750 -fold dilution, 0.20 ), in a total volume of $0.45 \mathrm{ml}$, and was preincubated at $30^{\circ} \mathrm{C}$ for $3 \mathrm{~min}$, and then $0.05 \mathrm{ml}$ of labeled phenylalanine or tyrosine (specific activity; $10 \mu \mathrm{Ci} / \mu \mathrm{mol}$ ) was added to a final concentration of $0.02 \mathrm{~mm}$. After incubation for $2.5 \mathrm{~min}, 0.1 \mathrm{ml}$ aliquots of the mixture were placed on membrane filters (Toyo TM-2, with a pore size of $0.45 \mu \mathrm{m}$ ) and then washed 8 times with $1 \mathrm{ml}$ of cold $0.01 \mathrm{M}$ Tris $-\mathrm{HCl}$ buffer, $\mathrm{pH} \mathrm{7.5}$. The radioactivity of the dried cells was measured.

Derivation of $m$ FP-resistant mutants. Strain No. 221-43 grown in Medium 41, and strain S-179 and No. 239 grown in Medium 7 supplemented with L-tyrosine and L-methionine $(200 \mathrm{mg} / \mathrm{l}$ each) for $16 \mathrm{hr}$ were harvested, washed once with $10 \mathrm{ml}$ of $0.1 \mathrm{M}$ sodium phosphate buffer, $\mathrm{pH}$ 7.0 , and then treated with 750,300 , and $750 \mu \mathrm{g} / \mathrm{ml}$ of $N$ methyl- $N^{\prime}$-nitro- $N$-nitrosoguanidine at $30^{\circ} \mathrm{C}$ for $15 \mathrm{~min}$ to give $19.5,1.0$, and $34 \%$ survivors, respectively. Then, the cells spread on selection agar plates containing Medium 41 supplemented with $300 \mathrm{mg} / 1$ of Tyr-Glu and $m \mathrm{FP}$ for strain No. 221-43, Medium 30 supplemented with $30 \mathrm{mg} / \mathrm{l}$ of monosodium glutamate, $200 \mathrm{mg} / \mathrm{l}$ of L-threonine, $200 \mathrm{mg} / \mathrm{l}$ of L-lysine, $50 \mathrm{mg} / \mathrm{l}$ of L-cysteine, $300 \mathrm{mg} / \mathrm{l}$ of TyrGlu and $m F P$ instead of tyrosine for strain S-179, and Medium 30 supplemented with $300 \mathrm{mg} / \mathrm{l}$ of Tyr-Glu and $m F P$ instead of tyrosine for strain No. 239. Colonies appearing during 8 to 10 days' incubation at $30^{\circ} \mathrm{C}$ were picked up as $m$ FP-resistant mutants. 
Production of L-phenylalanine or L-tryptophan. Mutants derived from strains No. 221-43, S-179, and No. 239 were aerobically cultured at $30^{\circ} \mathrm{C}$ for $72 \mathrm{hr}$ in $500 \mathrm{ml}$-flasks $(20 \mathrm{ml} / \mathrm{flask})$ or test tubes ( $3 \mathrm{ml} / \mathrm{tube})$ containing Medium 3, Medium 31, and Medium 31 but containing $500 \mathrm{mg} / 1$ of tyrosine, respectively. Phenylalanine and tryptophan were determined microbiologically as described previously. ${ }^{13}$.

\section{RESULTS AND DISCUSSION}

Competition in the uptake of aromatic amino acids and their derivatives

As shown in Fig. 2, the phenylalanine uptake was competitively inhibited by tyrosine,

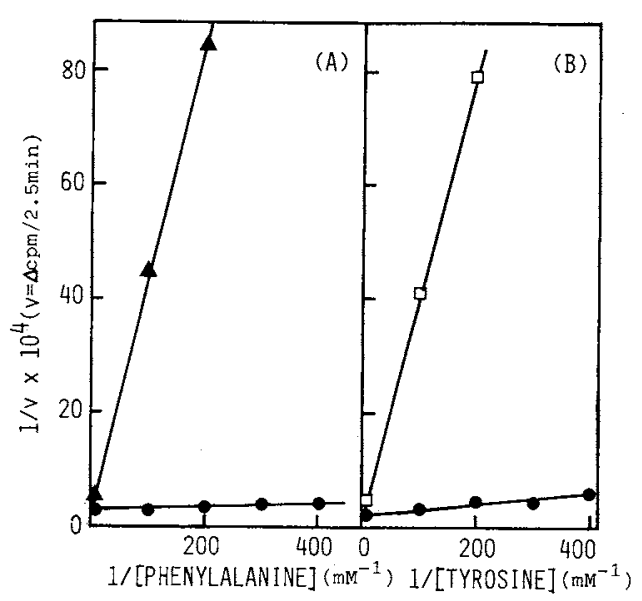

FIG. 2. Competitive Relationship in Cellular Transport Systems between Phenylalanine and Tyrosine.

The phenylalanine or tyrosine uptake was assayed under the standard conditions except for the phenylalanine or tyrosine concentration, respectively. To the assay mixture, $0.2 \mathrm{~mm}$ tyrosine $(\mathrm{A}, \boldsymbol{\Delta}), 0.2 \mathrm{~mm}$ phenylalanine $(\mathrm{B}, \square)$, or nothing (O) was added.

TABlE I. EFFECTS OF $m$ FP AND DiPEPTIDES Containing Tyrosine or Phenylalanine on Phenylalanine Uptake

\begin{tabular}{lcc}
\hline Addition & $\begin{array}{c}\text { Concentration } \\
(\mathrm{mM})\end{array}$ & $\begin{array}{c}\text { Relative uptake } \\
\text { rate }(\%)\end{array}$ \\
\hline None & - & $100^{\circ}$ \\
Tyrosine & 0.4 & 17 \\
$m$ FP & 1.0 & 9.8 \\
Tyr-Glu & 1.0 & 120 \\
Tyr-Ala & 1.0 & 111 \\
Phe-Ala & 1.0 & 127 \\
\hline
\end{tabular}

a $2951 \Delta \mathrm{cpm} / 2.5 \mathrm{~min}$. and the tyrosine uptake was competitively inhibited by phenylalanine in $B$. flavum, indicating that both are taken up into the cells with a common transport system. Table I also shows the inhibition of phenylalanine uptake by $m \mathrm{FP}$. This suggests that they are taken up by the common system. On the other hand, 3 tyrosine-containing dipeptides did not inhibit the phenylalanine uptake, suggesting that they were taken up systems other than that for phenylalanine, tyrosine, and $m \mathrm{FP}$. Furthermore, Tyr-Glu did not inhibit glutamate uptake (data not shown). A dipeptide transport system, distinct from those for its component amino acids, has been reported in Escherichia coli $^{15)}$ and Salmonella typhimurium. ${ }^{16)}$. These results indicate that $m F P$ competes in the cellular transport system with tyrosine, but not with Tyr-Glu. Therefore, it seems difficult to increase the intracellular concentrations by the addition of $m \mathrm{FP}$ and tyrosine to the medium, but it seems possible to do so by the addition of $m$ FP and Tyr-Glu. The Tyr-Glu uptake and formation of tyrosine from Tyr-Glu inside $B$. flavum cells were confirmed by the growth of a tyrosine auxotroph, S-179, in the presence of Tyr-Glu instead of the required amino acid, tyrosine. It showed maximum growth at $300 \mu \mathrm{g} / \mathrm{ml}$ of Tyr-Glu.

Sensitivities to phenylalanine analogues and effects of tyrosine-containing dipeptides

As shown in Fig. 3 (A), strain No. 221-43 having $\mathrm{PDT}^{\mathrm{R}}$ and feedback-sensitive $\mathrm{DS}$, a $m \mathrm{FP}$-resistant mutant derived from wild strain No. 2247 , was resistant to $m$ FP. That is, its growth was only inhibited $20 \%$ by $5000 \mu \mathrm{g} / \mathrm{ml}$ of $m \mathrm{FP}$. This resistance to $m \mathrm{FP}$ was not influenced by the addition of tyrosine, which synergistically inhibited DS with $m$ FP. However, the simultaneous presence of $m \mathrm{FP}$ and a tyrosine-containing dipeptide, Tyr-Clu or Tyr-Ala, which did not compete with $m \mathrm{FP}$ in the transport system, strongly inhibited the growth of the strain. The simultaneous presence of $m \mathrm{FP}$, tyrosine and glutamate did not make it sensitive to $m$ FP. Moreover, the growth inhibition by $m \mathrm{FP}$ and Tyr-Glu was 


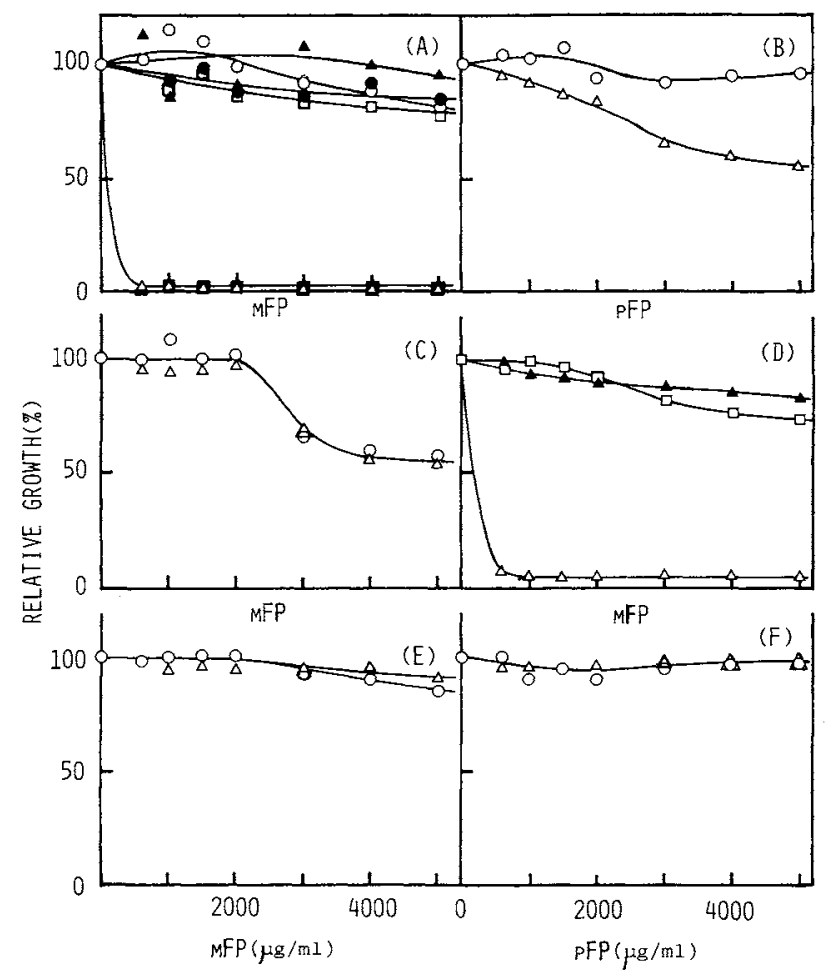

FIG. 3. Effects of Tyrosine-containing Peptides, $m \mathrm{FP}$, and $p \mathrm{FP}$ on the Growth of $\mathrm{PDT}^{\mathrm{R}}-, \mathrm{DS}^{\mathrm{R}}{ }_{-}$, or $\mathrm{DS}^{\mathrm{R}}$ PDT $^{R}$-Type Mutants.

Strains No. 221-43 (A and B), No. 482-18 (C), No. 239 (D), and M-145 (E and F) were cultured at $30^{\circ} \mathrm{C}$ for 16 , 40,24 , and $24 \mathrm{hr}$ in test tubes containing $5 \mathrm{ml}$ of Medium 41 with various additions, respectively. Relative growth was determined as the relative increase in absorbancy at $562 \mathrm{~nm}$ after 26 -fold dilution, as to that in the absence of $m \mathrm{FP}$ or $p \mathrm{FP}$. Nothing (O), $300 \mathrm{mg} / 1$ of Tyr-Glu $(\triangle), 300 \mathrm{mg} / \mathrm{l}$ of Tyr-Ala ( $\mathbf{\square}), 150 \mathrm{mg} / 1$ of tyrosine $(\square), 150 \mathrm{mg} / \mathrm{l}$ of tyrosine and $150 \mathrm{mg} / \mathrm{l}$ of monosodium glutamate $(0), 300 \mathrm{mg} / 1$ of Tyr-Glu and $200 \mathrm{mg} / 1$ of phenylalanine $(\mathbf{A})$, and the indicated concentrations of $m \mathrm{FP}$ or $p \mathrm{FP}$ were added to the medium. In (A), the absorbancies at $562 \mathrm{~nm}$ in the absence of $m \mathrm{FP}$ were $0.500(\mathrm{O}), 0.540(\triangle), 0.430(\mathbf{\square}), 0.500(\square)$, $0.540(\boldsymbol{O})$, and $0.500(\mathbf{A})$. In (D), $150 \mathrm{mg} / 1$ of methionine was further added.

completely recovered by the further addition of phenylalanine. Therefore the growth inhibition seems to be due to depression of phenylalanine biosynthesis through the synergistic inhibition of $\mathrm{DS}^{1)}$ by $m \mathrm{FP}$ and tyrosine formed from Tyr-Glu intracellularly.

$p \mathrm{FP}$, another phenylalanine analogue, which inhibits $\mathrm{PDT}^{2)}$ but not $\mathrm{DS}^{1)}$ with tyrosine synergistically, did not strongly inhibit the growth of strain No. 221-43 in the presence of Tyr-Glu, unlike $m$ FP (Fig. 3(B)). Furthermore, as shown in Fig. 3(C), the sensitivity to $m \mathrm{FP}$ of strain No. $482-18^{1)}$ having $\mathrm{DS}^{\mathrm{R}}$, another $m$ FP-resistant mutant derived from wild strain No. 2247, was not affected by the presence of Tyr-Glu. These results support that the growth inhibition in the presence of both $m \mathrm{FP}$ and Tyr-Glu was due to the inhibition of DS by $m \mathrm{FP}$ and tyrosine. The partial growth inhibition of strain No. 482-18 at high $m$ FP concentrations will be discussed later.

\section{Derivation of mutants having both $D S^{\mathrm{R}}$ and $P D T^{\mathrm{R}}$}

From the results described above, it seems that mutants having both $\mathrm{DS}^{\mathrm{R}}$ and $\mathrm{PDT}^{\mathrm{R}}$, which were suggested to accumulate larger amounts of phenylalanine than those having only $\mathrm{PDT}^{\mathrm{R}}$ or $\mathrm{DS}^{\mathrm{R}}$, can be selected by $m \mathrm{FP}$ - 
Table II. Phenylalanine and Tyrosine Production and Enzymatic Alteration in

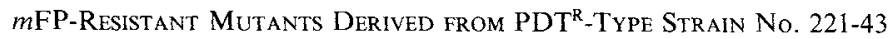

\begin{tabular}{ccccccc}
\hline \multirow{2}{*}{$\begin{array}{c}\text { Production } \\
(\mathrm{g} / 1)\end{array}$} & \multicolumn{5}{c}{ Parent and mutant strain No. } \\
\cline { 2 - 6 } & $221-43$ & $\mathrm{M}-51$ & $\mathrm{M}-144$ & $\mathrm{M}-145$ & $\mathrm{M}-154$ & M-211 \\
\hline L-Phe & 2.1 & 4.5 & 4.0 & 6.0 & 4.0 & 5.2 \\
L-Tyr & $<0.01$ & 0.14 & 0.11 & 1.4 & 0.22 & 0.11 \\
\hline
\end{tabular}

\begin{tabular}{|c|c|c|c|c|c|c|c|}
\hline \multicolumn{2}{|c|}{ Enzyme activity $(\%)$} & & & & & & \\
\hline Enzyme & $\begin{array}{l}\text { Addition } \\
\text { (1 mM) }\end{array}$ & & & & & & \\
\hline DS & None & $100(10.5)$ & $100(28.3)$ & $100(13.9)$ & $100(9.1)$ & $100(13.3)$ & $100(12.4)$ \\
\hline DS & Phe + Tyr & 29 & 94 & 91 & 93 & 99 & 90 \\
\hline $\mathrm{CM}$ & None & $100(2.1)$ & $100(0.78)$ & & $100(2.1)$ & & $100(1.3)$ \\
\hline & Phe & 9.7 & 280 & & 173 & & 242 \\
\hline & Tyr & 13 & 183 & & 148 & & 224 \\
\hline & $\operatorname{Trp}$ & 257 & 414 & & 218 & & 275 \\
\hline PDT & None & $100(2.6)$ & & & $100(4.2)$ & & $100(5.0)$ \\
\hline & Phe & 202 & & & 179 & & 210 \\
\hline & Tyr & 255 & & & 240 & & 313 \\
\hline & Trp & 101 & & & 100 & & 99 \\
\hline
\end{tabular}

The specific activities in $\mathrm{nmol} / \mathrm{min} / \mathrm{mg}$ protein are given in parentheses.

Abbreviations: Phe, phenylalanine; Tyr, tyrosine; Trp, tryptophan.

resistance in the presence of Tyr-Glu from among mutants derived from strains having $\mathrm{PDT}^{\mathrm{R}}$. After mutagenesis of strain No. 221-43, $m$ FP-resistant mutants were isolated as colonies appearing on agar plates containing 600 to $4000 \mu \mathrm{g} / \mathrm{ml}$ of $m \mathrm{FP}$ and $300 \mu \mathrm{g} / \mathrm{ml}$ of TyrGlu. Among 271 mutants, 14 accumulated more than $3.5 \mathrm{~g} / \mathrm{l}$ of phenylalanine in a test tube culture, in which the parent produced $2.0 \mathrm{~g} / \mathrm{l}$ of phenylalanine. The best production was $4.6 \mathrm{~g} / \mathrm{l}$ of phenylalanine. Table II shows the phenylalanine production of the best 5 strains in flask cultures after single colony isolation. The best producer, strain M-145, accumulated $6.0 \mathrm{~g} / 1$ of phenylalanine, that is, 2.9-times more than the parent strain. These strains accumulated tyrosine in addition to phenylalanine (Table II). Tyrosine accumulation is a characteristic of mutants having $\mathrm{DS}^{\mathrm{R}} \cdot 3$.

Enzyme alterations of these mutants were examined, as shown in Table II. All the strains tested had DSs resistant to synergistic inhibition by phenylalanine and tyrosine, and CMs resistant to inhibition by phenylalanine or tyrosine, in addition to $\mathrm{PDT}^{\mathrm{R}}$ inherited from the the parent. As reported previously, DS and CM form a bifunctional enzyme complex with common regulatory sites for phenylalanine and tyrosine. Therefore, the inhibitions of DS and CM are simultaneously removed on mutation. ${ }^{17}$ It is of interest enzymologically that phenylalanine and tyrosine, like tryptophan, stimulate the activity of feedback-resistant CMs.

Thus, mutants having both $\mathrm{PDT}^{\mathrm{R}}$ and $\mathrm{DS}^{\mathrm{R}}$ were obtained from strain No. 221-43 having only $\mathrm{PDT}^{\mathrm{R}}$. As shown in Fig. $3(\mathrm{E}, \mathrm{F})$, the sensitivities of strain $\mathrm{M}-145$ having $\mathrm{DS}^{\mathrm{R}}$ and $\mathrm{PDT}^{\mathrm{R}}$ to $m \mathrm{FP}$ and $p \mathrm{FP}$ were not affected by the addition of Tyr-Glu, in contrast to in the case of parent strain No. 221-43 having PDT $^{R}$. This result again supports that the growth inhibition of strains having $\mathrm{PDT}^{\mathrm{R}}$ alone by 
Table III. Phenylalanine and Tryptophan Production and Enzymatic Alteration in $m$ FP-Resistant Mutants Derived from Strains No. 239 and S-179

\begin{tabular}{lccccccccc}
\hline & \multicolumn{8}{c}{ Parent and mutant strain No. } \\
\cline { 2 - 8 } & 239 & M-87 & M-48 & M-245 & M-257 & M-128 & S-179 & M-423 \\
\hline L-Phe & 18.1 & 23.4 & 23.2 & 22.8 & 22.4 & 22.1 & 1.6 & 9.1 \\
L-Trp & - & - & - & - & - & - & 18.9 & 15.2 \\
L-Phe +L-Trp & & & & & & & 20.5 & 24.3 \\
\hline
\end{tabular}

Enzyme activity $(\%)$

\begin{tabular}{|c|c|c|c|c|c|c|c|c|c|}
\hline Enzyme & Addition (1 mM) & & & & & & & & \\
\hline \multirow[t]{2}{*}{ DS } & None & $100(16)$ & $100(18)$ & $100(19)$ & $100(15)$ & $100(12)$ & $100(18)$ & $100(47)$ & $100(54)$ \\
\hline & Phe + Tyr & 30 & 98 & 94 & 99 & 98 & 99 & 53 & 90 \\
\hline \multirow[t]{4}{*}{$\mathrm{CM}$} & None & $100(5.6)$ & $100(3.5)$ & $100(0.6)$ & $100(0.8)$ & $100(3.9)$ & $100(1.3)$ & $100(4.1)$ & $100(5.4)$ \\
\hline & Phe & 8.0 & 177 & 510 & 112 & 177 & 294 & 7.9 & 152 \\
\hline & Tyr & 15 & 143 & 286 & 194 & 140 & 182 & 12 & 123 \\
\hline & Trp & 161 & 213 & 928 & 356 & 217 & 547 & 189 & 205 \\
\hline
\end{tabular}

The specific activities in $\mathrm{nmol} / \mathrm{min} / \mathrm{mg}$ protein are given in parentheses.

- , not determined.

$m F P$ and Tyr-Glu is due to the synergistic inhibition of DS. It was also clarified that the weak growth inhbition by $p$ FP and Tyr-Glu of strain No. 221-43 was also caused by the DS inhibition and that the partial growth inhibition of strain No. $482-18$ by a high concentration of $m \mathrm{FP}$ was caused by the inhibition of PDT by $m$ FP.

\section{Application to the breeding of phenylalanine producers}

The method used for the derivation and selection of mutants having both $\mathrm{DS}^{\mathrm{R}}$ and PDT $^{\mathrm{R}}$ was applied for the improvement of phenylalanine producers. A phenylalanineproducing strain, No. 239, was used as the parent strain. In this strain, whereas DS was sensitive to the inhibition, similar to that in the wild strain (Table III), PDT with a specific activity of $0.9 \mathrm{nmol} / \mathrm{min} / \mathrm{mg}$ protein, was not inhibited at all but activated 7.7-or 10.9-times by $1.0 \mathrm{~mm}$ phenylalanine or tyrosine, respectively, the former of which completely inhibited PDT of the original wild strain, No. 2, with a specific activity of $0.4 \mathrm{nmol} / \mathrm{min} / \mathrm{mg}$ protein. As shown in Fig. 3(D), growth of strain No.
239 was almost completely inhibited by more than $600 \mu \mathrm{g} / \mathrm{ml}$ of $m$ FP in the presence of TyrGlu, similar to that of a simple $P D T^{R}$-type strain, No. 22l-43. Then, $1100 \mathrm{mFP}$-resistant mutants were isolated from agar plates containing 1000 to $4000 \mu \mathrm{g} / \mathrm{ml}$ of $m F P$ after independent mutagenizing treatment of strain No. 239 three times. Mutants producing more than $20 \mathrm{~g} / \mathrm{l}$ of phenylalanine in a test tube culture were obtained at a high frequency, for example, 33 out of 300 mutants in the second experiment. The production by the best 5 strains in flask cultures after single colony isolation is shown in Table III. The best production, $23.4 \mathrm{~g} / 1$ of phenylalanine, was shown by strain M-87, while the parent, No. 239 , produced $18.1 \mathrm{~g} / \mathrm{l}$. The DSs and CMs of these 5 mutants were resistant to the inhibition, similar to those derived from strain No. 221-43 (Table III). Thus, mutants having both $D^{R}$ and $P^{R} T^{R}$ could be derived from phenylalanine-producing strain No. 239 and the phenylalanine production was increased by $5.3 \mathrm{~g} / 1$ at maximum. 
Application to the breeding of tryptophanproducing mutants

The sulfaguanidine-resistant tryptophanproducing strain, S- 179 , used as the parent in this experiment was derived, similar to strain $\mathrm{S}-225,{ }^{6)}$ from strain A-100, ${ }^{8)}$ which is a 5fluorotryptophan- and azaserine-resistant tryptophan-producing mutant derived from a phenylalanine-producing strain, No. 239. Strain S-179 has PDT $^{\mathrm{R}}$ inherited from strain No. 239 and partially feedback-resistant DS inherited from strain A-100 (Table III). Since DS is a biosynthetic enzyme common to tryptophan and phenylalanine, complete removal of the feedback inhibition of DS could possibly lead to an increase of the tryptophan production. Since the growth of strain S- 179 was completely inhibited by more than $400 \mu \mathrm{g} / \mathrm{ml}$ of $m \mathrm{FP}$ in the presence of Tyr-Glu, $m$ FP-resistant mutants were isolated from agar plates containing 400 to $1000 \mu \mathrm{g} / \mathrm{ml}$ of $m \mathrm{FP}$. Contrary to our expectation, none out of the 481 mutants obtained showed higher productivity than the parent in a test tube culture. However, mutants showing higher phenylalanine accumulation were found. Strain M423 , selected from among them, accumulated larger total amounts of phenylalanine and tryptophan than the parent, as shown in Table III. The synergistic inhibition of DS by phenylalanine and tyrosine was almost completely removed in strain M-423 and the inhibitions of $\mathrm{CM}$ were also removed. Thus, a mutant having both $\mathrm{DS}^{\mathrm{R}}$ and $\mathrm{PDT}^{\mathrm{R}}$ could be derived from a tryptophan-producting strain, S-179, as in the cases of strain No. 221-43 and No. $239 . \mathrm{DS}^{\mathrm{R}}$ provided by this mutation seems to have increased the total amounts of tryptophan and phenylalanine accumulated, whereas the decreased tryptophan production and the increased phenylalanine production seem to be due to the feedback-resistant CM, because $\mathrm{CM}$ is the enzyme on the phenylalanine- side at the chorismate-branch point of the tryptophan and phenylalanine biosynthetic pathway. In order to remove the phenylalanine by-product, lacking of $\mathrm{CM}$ activity or complete removal of the feedback inhibition of anthranilate synthase at the tryptophanbranch seems necessary.

Acknowledgment. The authors are indebted to Dr. Y. Komachiya and Dr. R. Tsugawa for their encouragement during this work

\section{REFERENCES}

1) I. Shiio, S. Sugimoto and R. Miyajima, J. Biochem., 75, 987 (1974).

2) S. Sugimoto and I. Shiio, J. Biochem., 76, 1103 (1974).

3) S. Sugimoto, M. Nakagawa, T. Tsuchida and I. Shiio, Agric. Biol. Chem., 37, 2327 (1973).

4) S. Sugimoto and I. Shiio, Abstracts of Papers, 54th Annual Meeting of the Japanese Biochemical Society, Sendai, September, 1981, p. 984.

5) K. Sato, S. Kamata, T. Takenouchi, T. Tanaka and H. Enei, Japanese Patent Application, Kokai Sho 59. 34893 (1984).

6) I. Shiio, S. Sugimoto, and K. Kawamura, Agric. Biol. Chem., 48, 2073 (1984).

7) S. Sugimoto and I. Shiio, Agric. Biol. Chem., 49, 39 (1985).

8) I. Shiio, S. Sugimoto and K. Kawamura, Agric. Biol. Chem., 46, 1849 (1982).

9) I. Shiio and K. Ujigawa-Takeda, Agric. Biol. Chem., 44, 1897 (1980).

10) S. Sugimoto and I. Shiio, Agric. Biol. Chem, 46, 2711 (1982).

11) M. Mori and I. Shiio, Agric. Biol. Chem., 51, 129 (1987).

12) I. Shiio and K. Ujigawa, J. Biochem., 84, 647 (1978).

13) I. Shiio, S. Sugimoto and M. Nakagawa, Agric. Biol. Chem., 39, 627 (1975).

14) S. Sugimoto and I. Shiio, J. Biochem., 88, 167 (1980).

15) C. F. Higgins and M. M. Gibson, in "Methods in Enzymology," Vol. 125, ed. by S. Fleischer and B. Fleischer, Academic Press, New York, 1986, pp. $365 \sim 377$.

16) S.-L. Yang, J. Becker and F. Naider, Biochim. Biophys. Acta, 471, 135 (1977).

17) I. Shiio and S. Sugimoto, J. Biochem., 86, 17 (1979). 